ORIGINAL ARTICLE

\title{
fMRI-Driven DTT Assessment of Corticospinal Tracts Prior to Cortex Resection
}

\author{
Xiao-xiong Jia, Yang Yu, Xiao-dong Wang, Hui Ma, Qing-hua Zhang, \\ Xue-yin Huang, He-chun Xia
}

\begin{abstract}
Background: The role of diffusion tensor tractography (DTT) has become increasingly important in the preoperative mapping of brain white matter. Recently, functional magnetic resonance imaging (fMRI) driven DTT has provided the ability to evaluate the spatial relationship between the corticospinal tract (CST) and motor resection tumor boundaries. The main objective of this study was improvement of the preoperative assessment of the CST in patients with gliomas involving the motor cortical areas. Methods: Seventeen patients with gliomas involving motor cortical areas underwent 3 dimensions (3D) T1-weighted imaging for anatomical referencing, using both fMRI and diffusion tensor imaging (DTI). We used the fast-marching tractography (FMT) algorithm to define the $3 \mathrm{D}$ connectivity maps within the whole brain using seed points selected in the white matter adjacent to the location of fMRI activation. The target region of interest (ROI) was placed in the cerebral peduncle. Karnofsky performance status (KPS) scores were evaluated for each patient before and after surgery. Results: The CST of a total seventeen patients were successfully tracked by choosing seed and target ROI on the path of the fibers. What is more, DTT can indicate preoperatively the possibility for total glioma removal or the maximum extent of surgical resection. The postoperative average KPS score for the seventeen patients enrolled increased by more than 10 points. Conclusions: Incorporation of fMRI driven DTT showed a maximum benefit in surgical treatment of gliomas. Our study of the assessment precision should enhance the accuracy of glioma operations with a resulting improvement in postoperative patient outcome.
\end{abstract}

RÉSUMÉ: Tractographie en tenseur de diffusion réalisée en fonction de I'IRMf dans l'évaluation des faisceaux cortico-spinaux avant une résection du cortex moteur. Contexte : Le rôle de la tractographie en tenseur de diffusion (TTD) est devenu de plus en plus important dans la cartographie préopératoire de la substance blanche. Grâce à la tractographie en tenseur de diffusion réalisée en fonction de l'IRM fonctionnelle (IRMf) nous pouvons maintenant évaluer la relation spatiale entre le faisceau pyramidal (FP) et les limites de la tumeur. L'objectif principal de cette étude était d'améliorer l'évaluation préopératoire du FP chez les patients présentant un gliome dans la région du cortex moteur. Méthode : Dix-sept patients atteints d'un gliome impliquant le cortex moteur ont subi une imagerie tri-dimensionnelle pondérée en T1 à des fins de référence anatomique, au moyen de l'IRMf et de la TTD. Nous avons utilisé l'algorithme de tractographie rapide pour définir des cartes de connectivité 3D dans tout le cerveau au moyen de points de référence choisis dans la substance blanche adjacente au site de l'activation observée à l'IRMf. La zone-cible d'intérêt (ZCI) était située dans le pédoncule cérébral. L'indice de performance de Karnofsky (KPS) était évalué chez chaque patient avant et après la chirurgie. Résultats : Le FP a été tracé avec succès chez dix-sept patients en choisissant des points de référence et des cibles ZCI sur le trajet des fibres. De plus, la TTD peut renseigner avant l'opération sur la possibilité d'une exérèse complète du gliome ou l'étendue maximale de la résection chirurgicale possible. Le score KPS moyen postopératoire chez les 17 patients de l'étude a augmenté de plus de 10 points. Conclusions : L'ajout de la TTD réalisée en fonction de l'IRMf a procuré un bénéfice très important dans le traitement des gliomes. Notre étude de l'évaluation de sa précision devrait rehausser la précision dans la chirurgie du gliome et ainsi améliorer le résultat postopératoire chez ces patients.

Can J Neurol Sci. 2013; 40: 558-563

Neurosurgical treatment of gliomas involving the eloquent cortical areas has, as its primary goal, the resection of the maximum amount of tumor while still preserving the critical cortices of the brain in order to enhance patient postoperative quality of life. Recently, blood-oxygen-level-dependent (BOLD) functional magnetic resonance imaging (fMRI) has been proven to be able to identify the cortical primary motor areas (PMA) ${ }^{1,2}$. However, the question of brain surgery in the vicinity of important white matter tracts remains problematic, such as corticospinal tract (CST) deformation by gliomas involving the motor cortex. Inadvertent transection of the CST may result in devastating neurologic damage to the white matter either by disruption, displacement or deformation of the fibers ${ }^{3-5}$. Therefore, visualization of the different components of the CST should increase a neurosurgeon's understanding of the brain

From the Department of Neurosurgery (XXJ, YY, HM, QHZ, HCX), Department of Radiology (XDW, XYH), General Hospital of Ningxia Medical University, Yinchuan, China.

Received October 19, 2012. Final Revisions Submitted January 4, 2013. Correspondence to: He-chun Xia, Department of Neurosurgery, General Hospital of Ningxia Medical University, Yinchuan, 750004, China. Email:xhechun@yahoo.com.cn 
anatomy and provide important additional information for enhanced preoperative tumor removal assessment $t^{6,7}$.

However, fMRI can't provide useful information regarding the important subcortical and deep white matter tracts, including CST fibers. Diffusion tensor tractography (DTT) can visualize the major white matter tracts in three dimensions (3D) by setting region of interest (ROI) on the path of the fibers using BOLD imaging for delineation of the eloquent $\operatorname{cortex}^{8,9}$. Until now, these ROIs have almost exclusively been chosen on the basis of anatomic landmarks using diffusion tensor imaging (DTI) 2Dbased fractional anisotropy (FA) maps. Diffusion tensor tractography of a CST based solely on its assumed anatomic location, such as the presumed location of the PMA or the cerebral peduncle, is not sufficient for optimal patient outcomes $^{10-13}$. In addition, the tracking of fibers adjacent to a glioma is further complicated due to changes in the diseased tissue, such as edema, tissue compression and degeneration. Thus, the fibers cannot be distinguished from local brain structures when the PMA and CST are affected by the glioma and surrounding edema ${ }^{14-17}$. These changes may deform the position of the seed ROI to show the architecture of the white matter and its most important subcomponents. Therefore, an accurate definition of seed and target regions for the reconstruction of the CST fiber pathway becomes even more challenging in patients suffering from tumors due to the displacement of the tissue and the distortion of anatomical landmarks around the lesion ${ }^{18,19}$. We used a fast-marching tractography (FMT) algorithm to define 3D connectivity maps within the whole brain employing seed points selected in the white matter adjacent to the location of maximum fMRI activation $^{20}$. By using the fMRI activations as landmarks for the seed ROI selection, we were able to track the complete path of the fiber system in situations when the fibers were displaced. Following the identification of the functional fiber system, we could then specifically characterize the effect of the glioma on the affected white matter.

The purpose of our study was to prospectively evaluate whether preoperative fMRI-driven DTT could be used to show a maximal benefit for precise visualize of the spatial relationship between the CST and glioma. What is even more important, the relationship between the increasing amplitude of the number of effective fibers at affected sites and the patients' Karnofsky performance status (KPS) at six months was addressed. In this study, we confirmed the usefulness of this advanced technique to precisely improve the preoperative schedule, decrease motor deficits and enhance the accuracy of the post-surgical patient outcome.

\section{Materials And Methods}

\section{Patients}

Seventeen patients with cerebral gliomas involving motor cortical areas were included in this study from December 2007 to October 2011 in the General Hospital of Ningxia Medical University. The exclusion criteria were as follows: patients with secondary or recurrent gliomas, patents with more than one operation, and patients for whom initial muscle strength grade of the affected extremities was less than 2/5 (full range of motion without gravity). The ages of the patients ranged from 27-67 years-old with an average age of 50.5 years. Among the 17 patients enrolled there were 6 men and 11 women.

Of the 17 subjects eligible for surgery based on BOLD and DTT evaluations, histopathologic examination confirmed the presence of a malignant tumor. Clinical information on the patients' pre- and postoperative neurological status was obtained from medical records and neurosurgical reports. According to the World Health Organization (WHO) classification for tumors of the nervous system (2007) pathology grading, the glioma tissues in our study included 13 astrocytomas, two oligodendrogliomas, two glioblastoma. The characteristics of the patients are shown in the Table.

\section{fMRI Data Acquisition and Analysis}

Imaging was performed on a Signal Excite HD 3.0T (General Electric company) magnetic resonance imaging (MRI) system. For anatomical reference, we used a 3D T1-weighted, spoiled gradient echo sequence recalled acquisition sequence consisting of 248 interleaved slices in axial orientation with the following parameters: repetition time $(\mathrm{TR})=20 \mathrm{~ms}$; echo time $(\mathrm{TE})=$ $2.4 \mathrm{~ms}$; acquisition matrix, $288 \times 256$ pixels; field of vision (FOV) $=240 \times 240 \mathrm{~mm}^{2} ;$ slice thickness $=1.2 \mathrm{~mm}$; flip angle $13^{0} ; \mathrm{NEX}=$ 1. We used gradient echo planar imaging (GRE-EPI) for BOLD image collection. The imaging parameters were as follows: $\mathrm{TR}=$ $3000 \mathrm{~ms}$; TE $=35 \mathrm{~ms}$; acquisition matrix, $64 \times 64$ pixels; FOV $=$ $240 \mathrm{~mm} \times 240 \mathrm{~mm}^{2}$; flip angle $90^{\circ} ; \mathrm{NEX}=1$. Five-millimeter

Table: General information, location of tumor and KPS score

\begin{tabular}{ccccc}
\hline \multirow{2}{*}{ No. } & \multirow{2}{*}{ Age/gender } & Location of tumor & \multicolumn{2}{c}{ KPS score } \\
\cline { 4 - 5 } & & pre & post \\
\hline 1 & $61 / \mathrm{M}$ & Left frontal & 90 & 100 \\
2 & $52 / \mathrm{F}$ & Right frontoparietal & 60 & 90 \\
3 & $66 / \mathrm{F}$ & Left frontal & 80 & 90 \\
4 & $37 / \mathrm{F}$ & Right frontal & 70 & 80 \\
5 & $56 / \mathrm{F}$ & Left parietal & 70 & 80 \\
6 & $44 / \mathrm{M}$ & Left frontal & 90 & 100 \\
7 & $67 / \mathrm{F}$ & Right frontal & 80 & 90 \\
8 & $40 / \mathrm{M}$ & Left frontal & 60 & 70 \\
9 & $47 / \mathrm{F}$ & Left frontoparieta & 80 & 90 \\
10 & $67 / \mathrm{M}$ & Left frontal & 90 & 100 \\
11 & $61 / \mathrm{M}$ & Left frontal & 90 & 90 \\
12 & $42 / \mathrm{F}$ & Right frontal & 60 & 70 \\
13 & $34 / \mathrm{F}$ & Left frontal & 90 & 90 \\
14 & $27 / \mathrm{F}$ & Right frontoparieta & 70 & 80 \\
15 & $56 / \mathrm{F}$ & Right parietal & 70 & 80 \\
16 & $49 / \mathrm{M}$ & Left temporoparietal & 90 & 100 \\
17 & $52 / \mathrm{F}$ & Right frontal & 80 & 90 \\
\hline & & & & \\
\hline
\end{tabular}


thickness sections were obtained and the acquisition time was 324 seconds. Echo planar images were performed by the software package SPM8.

Blood-oxygen-level-dependent fMRI mapping of all patients was successful. Finger tapping was performed either with the right or the left hand separately, or with both hands simultaneously, and consisted of self-paced consecutive opposition of the thumb to each of the other fingers of the hand ${ }^{21}$. Apart from the initial rest lasting 24 seconds, each fMRI acquisition consisted of five blocks of 30 seconds (s) motor activity alternating with five blocks of 30 s rest, resulting in a total acquisition time of 324 seconds. Subjects were instructed to perform the tasks consciously and to limit motion of their forearm, upper arm and other parts of their body. After the images were acquired, data were transferred to the Matlab workstation for analysis. Statistical analysis of fMRI data was performed using a t-test, with corrected values of $\mathrm{P}<0.05$ considered statistically significant by using SPM8 in Matlab.

\section{DTT Data Acquisition and Analysis}

The DTI data acquisition sequence was a spin echo-echo planar imaging (SE-EPI) sequence with $\mathrm{TR}=6000 \mathrm{~ms}$; $\mathrm{TE}=$ $77 \mathrm{~ms}$; acquisition matrix, $128 \times 128$ pixels; FOV $=240 \mathrm{~mm} \times$ $240 \mathrm{~mm}^{2}$; slice thickness $=5.0 \mathrm{~mm}$. Diffusion weighting with a maximal $\mathrm{b}$ factor of $1,000 \mathrm{~mm}^{2} / \mathrm{s}$ was carried out along 21 noncollinear directions. The DTI acquisition time was 276 seconds. All the DTI related calculations, the subsequent fiber tracking, and the statistical evaluation of the fiber tracks were performed using a dedicated Trackvis software package.

Diffusion tensor imaging data were processed and analyzed off-line with the Trackvis tool by the FMT algorithm method. Tracking was initiated in 3D-seed-areas computed on the basis of the white matter area immediately subjacent to fMRI activity in the precentral cortical gray matter, and the entire cerebral peduncle was chosen as the target region of interest ${ }^{22}$. The FA stop criterion was defined as 0.18 . Both the DTI and the fMRI data were loaded into the software package, which enabled us to perform seed ROI selection driven by the locations of the coregistered fMRI activations. Finally, only those traces that passed through common start and end points were displayed and were considered to be part of the CST.

\section{Results and Neurosurgical Procedures}

In all 17 admitted patients, we were able to track the hand fibers of the CST from all PMA by using the fMRI-based seed ROI approach. In the patient data using this new method, the CST fibers corresponded with the results from the KPS evaluation for the patients' pre-and postoperately.

As a representative example, a 47-year-old woman presented with a one-month history of progressive unsteadiness of gait and right-side weakness. Neurological examination showed decreased power on the right upper limb (4/5) and lower limb $(3 / 5)$. There were no cerebellar signs nor paresis of the right extremities. Conventional MRI showed a hypo-intense lesion on $\mathrm{T} 2-\mathrm{WI}$ in the left frontoparietal cortical and subcortical region, which had marked enhancement after contrast (Figure 1A and B). There was extensive perilesional edema with the resultant mass effects distorting the normal anatomy.

Before fiber tracking, we defined the FA threshold value as 0.18 for tracking fibers. Two ROIs were put on each side of the images: the cerebral peduncle was chosen as a target ROI and the apex of the activation areas was selected as a seed ROI (Figure $1 \mathrm{C}$ and $\mathrm{D})$. The final histological diagnosis was infiltrating astrocytoma (WHO grade III). In these subject data sets, the hemispheric location of the tracking seed areas was quite asymmetric due to the space occupying effect of the tumor and peritumoral edema. This preoperative information was useful to the surgeon and was used in planning the surgery with gross total

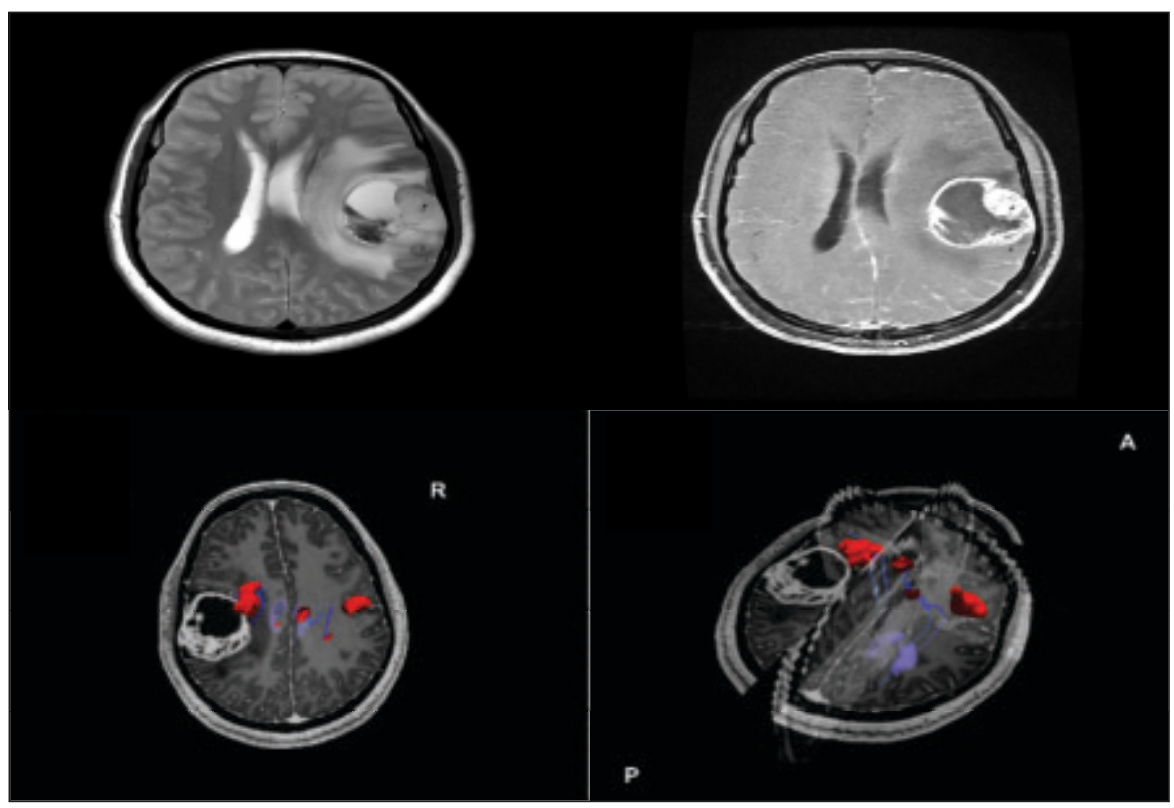

Figure 1: A 49-year-old woman with highgrade glioma with motor area involvement experienced onset of right hand and leg weakness one month before surgery. Conventional magnetic resonance imaging scans revealed a hypo-intense lesion on T2-WI in the left frontoparietal cortical and subcortical region (A), which had marked enhancement after enhancement (B). The reconstruction of DTI images constructed from both left and right tracts before surgery $(C, D)$. Panel $C$ and $D$ show fiber tracking results based on fMRI landmarks (red areas on the $3 D$ volume) on both side and top brain views. At the current state of the art, the combination of these figures shows the right tract less than the fibers of contralateral preoperatively. With this procedure, the CST seems to give a more complete picture of significant displacement of the fibers superiorly and inferiorly by the lesion and some peritumoral edema. 
resection of the tumor. The detected activation areas and the tracking fibers corresponded with the results from intraoperative electro-stimulation (IES). The patient showed no motor weakness or tumor progression during her six-month follow-up analysis.

In all patients, the PMA cortical region, as shown by fMRI and the observed tracking CST fibers were displaced by the tumor compared with the other side. As indicated by BOLD, functional cortices were confirmed by cortical IES ${ }^{23}$. Based on our protocol, we defined the boundary for surgical resection as the contiguous region consisting of the glioma resectional boundary. We evaluated our patients based on the study plan and decided the need and extent of coverage intraoperatively. Historically, the safe distance between the resected cortex border and eloquent area is at least $10 \mathrm{~mm}^{24}$. This safe distance is considered the "gold standard" for identification of critical areas and was spared from resection with a margin of at least $10 \mathrm{~mm}$ from the resection boundary. The critical auditory naming cortex and visual naming (VN) cortex were spared, with at least a $10 \mathrm{~mm}$ margin from the resection boundary ${ }^{25-28}$.

However, the boundaries were modified, if necessary, by the presence of eloquent cortex in the activity area as determined by functional stimulation. During fiber tracking, if start regions were slightly shifted in the activation area chosen as a ROI, the combined fMRI and DTT approach can prevent the problem of estimating completely different fiber systems, which might belong to the target system. The surgical approach was made based on the results of the integration of BOLD data and DTT mapping to fully expose the functional cortices.

The mean cortical electro-stimulation for central sulcus was then compared with the mapping of the PMA. The comparative results were in accordance with the postoperative KPS scores.

No obvious harmful effects to the sensory and motor cortex were observed. The postoperative average KPS score for the seventeen successfully admitted patients increased by more than ten points (Table). The KPS score before and after the operation were compared by the paired-sample t test. A P value of less than 0.01 was considered statistically significant.

\section{Discussion}

In our study, we showed that tracking of the CST directly from the fMRI activation area could be used to visualize and distinguish the different components of the CST.

The goal of the preoperative study was to provide more precise information of the spatial relationship between the nerve fibers and the lesions. Tracts were classified as unchanged, displaced, or infiltrated. The presence of intact fibers was predictive of better surgical outcomes because these cases showed a higher probability of total resection than did subtotal and partial resection. To make a distinction between these regions with tumor, conventional tractography has been applied, however, this information cannot be obtained from conventional fMRI imaging or from conventional DTT based on anatomic landmarks ${ }^{29,30}$. In some of these cases due to brain pathology such as gliomas, the white matter fibers are displaced, thus exposing the conventional tractography results to artifacts caused by this common analysis procedure. This approach used seed ROI selection, based on known anatomical landmarks, according to a DTI white matter atlas. Finding the route of the displaced fibers by utilizing trial and error might be successful in some cases, but, at the same time, is highly prone to subjective decisions made by the analyzer. This trial and error method also includes tampering with affected ROI, which might lead to erroneous fiber tracking, especially for tumors involving the seed ROI. In the presence of distorted anatomy, the surgeon must employ greater care and additional information is necessary.

Therefore, our study prospectively provided additional essential information not only on the location of motor cortical areas but also on the course of important subcortical white matter tracts during the preoperative assessment of patients with brain tumors. In patients with a brain tumor, the course of the white matter tract and its relationship to the tumor may be delineated by our integrated fMRI-based DTT method. This is especially relevant for deep-seated or subcortical lesions that distort the normal brain anatomy. Tractography of the CST fiber was hampered in the cases of anatomic distortion due to a mass effect of the lesion or in cases of altered diffusivity due to tumor infiltration or peritumoral edema in the region of the fibers. Tracking improved when the fMRI-based seed ROI approach was used, thus providing more reliable preoperative information. Before fiber tracking, DTT was depicted with a DTI analyzer using a 2-ROI method, with a target point manually set at the cerebral peduncle and the PMA used as a seed point for activation. In order to define the tracking seed regions, a center of gravity approach weighted with a significance level was applied in this study ${ }^{31}$. We would delineate the normal unaffected brain tissue from the fibers infiltrated by tumor, whereby optimal tumor resection is performed with minimal damage to vital brain connection functions (Figure 2). This distinction may provide additional presurgical information, in particular, allowing a more specific risk estimate before neurosurgical resection of a lesion as well as guiding electric stimulation of the subcortical white matter intraoperatively. We were able to characterize the changes in the white matter tract as displacement by the tumor adjacent to the CST fibers.

Resection of intracranial gliomas requires a detailed understanding of the fiber structure as well as functional anatomy of the tumor and adjacent brain tissue. This is extremely important in tumors involving motor pathway regions, especially for cases in which the PMA is located away from the tumor, but the CST runs close to the tumor area. A further strength of our study lies in the clinical applicability of our protocol. The presence of infiltrated or displaced CST was predictive of a lower probability of total resection. Patients with multifocal lesions have at least some edema, but we could also obtain reliable reconstruction of tracts through regions of tumor edema because of the small amount of the edematous lesions. In fact, the presence of the less edematous lesions was predictive of a higher probability of total resection. The mapping could contribute to a more accurate selection of a therapeutic strategy in cerebral gliomas. However, with the presence of infiltrated/ disrupted fibers, we introduced a strategy of a planned series of fractionated resections to maximize safe tumor removal without inducing permanent functional deficits. The fMRI-based DTT of the CST was effectively able to foresee the possibility of achieving a partial resection instead of a total resection, both for smaller tumors and for large lesions located in the motor pathway or subcomponent area, when surgery was performed for 


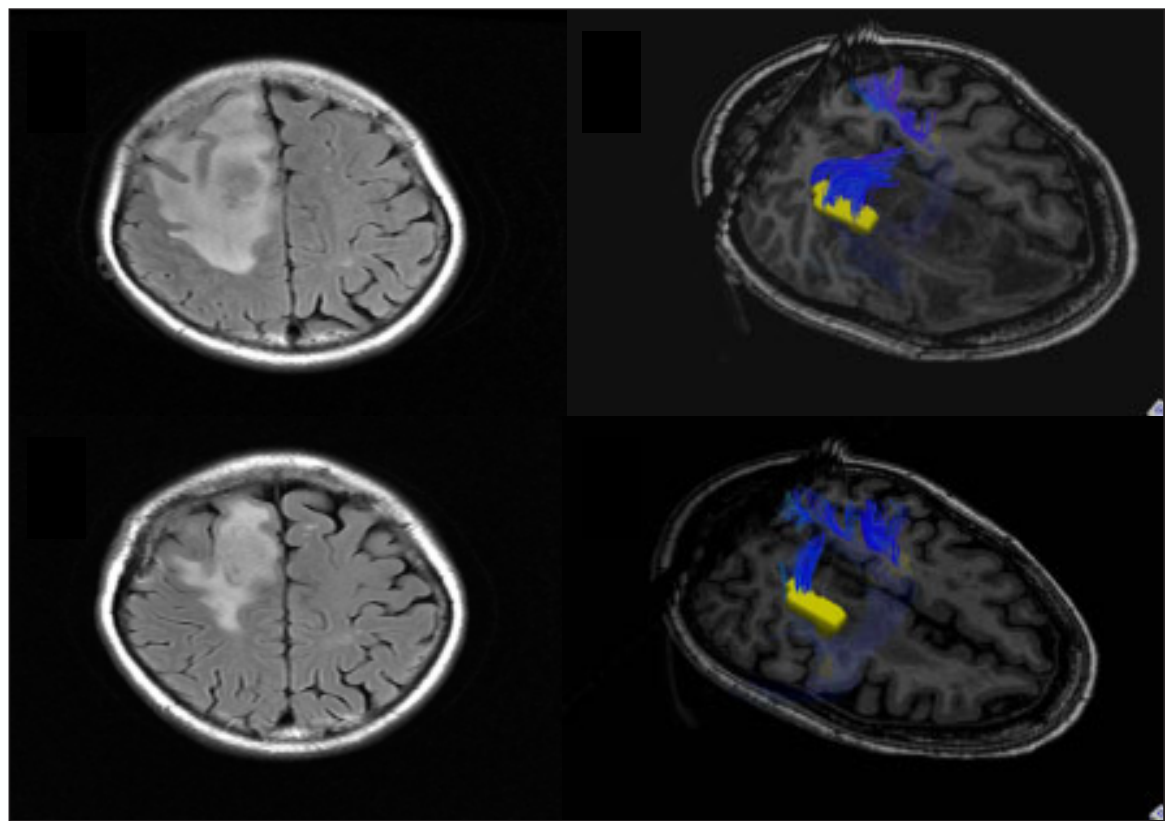

Figure 2: A 67-year-old woman with gliosarcoma and heavy peritumoral edema involving the motor area and CST (A). The fiber tracking of CST (blue areas) based on fMRI landmarks (yellow areas on the $3 D$ volume) provided independent and complementary information pre-and postoperatively. Before surgery, fMRI-based DTT imaging showed the right motor cortex was displaced anterolaterally by the tumor mass and edema. The images also revealed the ipsilateral CST originating from the motor cortex area posterior to the tumor and passing through the cerebral peduncles down to the lower brainstem. The CST was clearly displaced posteriorly by the mass effect $(B)$. During the operation procedure, the tumor was resected partly and the edema had almost disappeared (C). Panel D shows fiber tracking results combined with fMRI after surgery. At the current state of the art, the CST seems to be revealing a significant shift of the fibers posteromedially by the resection.

functional limits. What is more important, the integration of both high resolution anatomic data with fMRI data in the fiber tracking procedure made an evaluation of the spatial relationship between tracked fibers and tumor borders possible.

In addition, this study evaluated these effects by providing the IES based on the accurate identification of eloquent areas chosen as seed ROI and safely maintaining a high rate of functional preservation. The tumor and central sulcus were exposed in patients after craniotomy. The mean IES for central sulcus was then compared with the mapping of the PMA; The comparative results correlated with the postoperative KPS scores.

The importance of preserving function during glioma surgery aims to enhance patient quality of life. There are a number of techniques utilizing fMRI, direct electrophysiological monitoring and functional neuronavigation to maximize and safely resect gliomas. Neuronavigation is an extremely valuable tool and adds to the neurosurgeon's armamentarium. Combining these technologies should enhance the safety and efficacy for glioma surgery. These were the desired endpoints for our study where we successfully combined both DTT and subcortical stimulation using a single probe to safely resect a recurrent glioma. Future development should aim at expanding the range of applications beyond the limit of motor areas. The range of applications would then not be limited to the motor system, which was used exemplarily to describe this new method. In the future, the sensory, visual, and semantic systems will be used to further establish this method.

\section{Conclusions}

We proposed a clinically feasible protocol of integrating BOLD and DTT to optimize the extent of resection involving the cortical motor areas and subcortical white matter tracts in patients with gliomas in the brain. Particularly, the involvement of the intact fibers was a strong predictor for allowing a total tumor resection or the greatest extent of surgical removal of the preoperative tumor. Additionally, we introduced a strategy of fractionated resections to maximize a safe tumor removal process involving the presence of infiltrated or displaced fibers in the gliomas. Accordingly, this information should help neurosurgeons resect the maximum amount of tumor while still preserving the most critical cortices of the brain, thus resulting in enhanced postoperative quality of life for their patients.

\section{ACKNOWLEDGEMENTS}

This work was supported by a grant from the Natural Science Foundation of China (NO. 81260373) and Technological Project of Ningxia (NO. 2011-25).

\section{REFERENCES}

1. Sunaert S, Yousry TA. Clinical applications of functional magnetic resonance imaging. Neuroimaging Clin N Am. 2001;11(2): 221-36.

2. Haughton VM, Turski PA, Meyerand B, Wendt G, Moritz CH, Ulmer J. The clinical applications of functional magnetic resonance imaging. Neuroimaging Clin N Am. 1999;9(2): 285-93.

3. Werring DJ, Clark CA, Parker GJM, Miller DH, Thompson AJ, Barker GJ. A direct demonstration of both structure and function in the visual system: combining diffusion tensor imaging with functional magnetic resonance imaging. Neuroimage. 1999;9(3): 352-61.

4. Holodny AL, Ollenschleger MD, Liu WC, Schulder M, Kalnin AJ. Identification of the corticospinal tracts achieved using bloodoxygen-level-dependent and diffusion functional magnetic resonance imaging in patients with brain tumors. AJNR Am J Neuroradiol. 2001;22(1):83-8.

5. Schonberg T, Pazit P, Hendler T, Pasternak O, Assaf Y. Characterization of displaced white matter by brain tumors using combined diffusion tensor imaging and functional magnetic resonance. Neuroimage. 2006;30(4):1100-11. 
6. Smits M, Vernooij MW, Wielopolski PA, Vincent AJ, Houston GC, van der Lugt A. Incorporating functional magnetic resonance imaging into diffusion tensor tractograghy in the preoperative assessment of the corticospinal tract in patients with brain tumors. AJNR Am J Neuroradiol. 2007;28(7):1354-61.

7. Qiu TM, Zhang Y, Wu JS, et al. Virtual reality presurgical planning for cerebral glioma adjacent to motor pathways in an integrated 3 -D stereoscopic visualization of structural magnetic resonance imaging and diffusion tensor imaging tractography. Acta Neurochir. 2010;152(11):1847-57.

8. Mori S, van Ziji PC. Fiber tracking: principles and strategies a technical review. NMR Biomed. 2002;15(7-8):468-80.

9. Mori Masutani Y, Aoki S, et al. Simple visualization of the corticospinal pathway using tractography: one-roi and two-roi methods. Nippon Acta Radiologica. 2003;63(1):51-3.

10. Holodny AI, Gor DM, Watts R, Gutin PH, Uluq AM. Diffusiontensor magnetic resonance tractography of somatotopic organization of corticospinal tracts in the internal capsule: initial anatomic results in contradistinction to prior reports. Radiology. 2005;234(3):649-53

11. Catani M, Howard RJ, Pajevic S, Jones DK. Virtual in vivo interactive dissection of white matter fasciculi in the human brain. Neuroimage. 2002;17(1):77-94.

12. Akai H, Mori H, Aoki S, et al. Diffusion tensor tractography of gliomatosis cerebri: fiber tracking through the tumor. J Comput Assist Tomogr. 2005;29(1):127-9.

13. Stieltjes B, Kaufmann WE, van Zijl PC, et al. Diffusion tensor imaging and axonal tracking in the human brainstem. Neuroimage.2001;14(3):723-35.

14. Tao XF, Wang ZQ, Gong WQ, Jiang QJ, Jiang QJ, Shi ZR. A new study on diffusion tensor imaging of the whole visual pathway fiber bundle and clinical application. Chinese Med J. 2009;122 (2): $178-82$

15. Wu CX, Pu S, Lin Y, Wang YZ, et al. Fractionated resection on low grade gliomas involving Broca's area and insights to brain plasticity. Chinese Med J. 2008;121(20):2026-30.

16. Pradeep NM, Zhang S, Todd WV. Ictal high-frequency oscillations in neocortical epilepsy: implications for seizure localization and surgical resection. Epilepsia. 2011;52(10):1792-801.

17. Dorothee S, Bjorn WK, Susanne S, et al. Ventral and dorsal pathways for language. PNAS of the USA. 2008;105(46): $18035-40$

18. Guye M, Parker GJ, Symms M, et al. Combined functional magnetic resonance imaging and tractography to demonstrate the connectivity of the human primary motor cortex in vivo. NeuroImage. 2003;19(4):1349-60.
19. Krishnan R, Raabe A, Hattingen E, et al. Functional magnetic resonance imaging-integrated neuronavigation: correlation between lesion-to-motor cortex distance and outcome. Neurosurgery. 2004;55(4):914-5

20. Ludemann L, Forschler A, Grieger W, Zimmer C. Bold signal in the motor cortex shows a correlation with the blood volume of brain tumors. J Magn Reson Imaging. 2006;23(4):435-43.

21. Corie W, Wei GG, Mikulis DJ. Tumor effects on cerebral white matter as characterized by diffusion tensor tractography. Can J Neurol Sci. 2007;34(1);62-9

22. Holodny AI, Watts R, Korneinko VN, et al. Diffusion tensor tractography of the motor white matter tracts in man: current controversies and future directions. Ann N Y Acad Sci. 2005; 1064:88-97.

23. Field AS, Alexander AL, Wu YC, Hasan KM, Witwer B, Badie B. Diffusion tensor eigenvector directional color imaging patterns in the evaluation of cerebral white matter tracts altered by tumor. J Magn Reson Imaging. 2004;20(4):555-62.

24. Ozawa N, Muragaki Y, Nakamura R, Iseki H. Identification of the pyramidal tract by neuronavigation based on intraoperative diffusion-weighted imaging combined with subcortical stimulation. Stereotact Funct Neurosurg. 2009; 87(1):18-24.

25. Marla JH, William TS, Guy MM, Perrine K, Goodman RR. Brain stimulation reveals critical auditory naming cortex. Brain. 2005; 128(11):2742-9.

26. Lo CY, Chao YP, Chou KH, Guo WY, Su JL, Lin CP. DTI-based virtual reality system for neurosurgery. Conf Proc IEEE Eng Med Biol Soc. 2007;2007:1326-9.

27. Sanai N, Berger M. Glioma extent of resection and its impact on patient outcome. Neurosurgery. 2008;62(4):753-64.

28. Satoshi M, Masazumi F, Norimoto N, et al. Clinical indications for high-field $1.5 \mathrm{~T}$ intraoperative magnetic resonance imaging and neuro-navigation for neurosurgical procedures. Neurol Med Chir (Tokyo). 2009;49(8):349-50.

29. Baird AA, Colvin MK, Vanhorn JD, Inati S, Gazzaniga MS. Functional connectivity: interating behavioral, diffusion tensor imaging, and functional magnetic resonance imaging data sets. J Cogn Neurosci. 2005;17(4):687-93.

30. Bello L, Gambini A, Castellano A, et al. Motor and language DTI fiber tracking combined with intraoperative subcortical mapping for surgical removal of gliomas. Neuroimage. 2008;39(1): $369-82$.

31. Raimund K, Philipp S, Anton V, Boesiger P, Kollias S. Impact of fMRI-guided advanced DTI fiber tracking techniques on their clinical applications in patients with brain tumors. Neuroradiology. 2010; 52(1):37-46. 